\title{
INSTABILITIES \\ OF A THERMO-MECHANO-CHEMICAL SYSTEM
}

$\mathrm{BY}$

\author{
XANTHIPPI MARKENSCOFF
}

Dept. of Mechanical and Aerospace Engineering, University of California, San Diego, La Jolla, CA

Abstract. A Hadamard instability analysis of the partial differential equations governing a coupled thermo-mechano-chemical system predicts instability of the spinoidal decomposition type as well as instability of the "negative creep" type.

I. Introduction. The well-known instability of a chemical system is the CahnHilliard [1] spinoidal decomposition. However, in a thermo-mechano-chemical system more instabilities may occur. "Negative creep", a term introduced by Li [2], has been observed [3, 4] implying negative elastic moduli of the material. Li attempted to explain the phenomenon ad hoc in analogy to chemical spinoidal decomposition. Here we present a full instability analysis of the system of partial differential equations governing a thermo-mechano-chemical system. Constitutive equations for such systems have been developed by Bowen [5], Larché and Cahn [6], and others. We present a Hadamard instability analysis for a one-dimensional thermo-mechano-chemical system. In addition to the chemical spinoidal decomposition type of instability, a "negative creep" type is also shown to occur. Special cases when the thermal conductivity coefficient or the diffusion coefficient vanish are also examined.

II. Governing field equations for a thermo-mechano-chemical system. The governing field equations for a thermo-mechano-chemical system are:

$$
\begin{aligned}
\frac{\partial^{2} w}{\partial t^{2}}-\frac{\partial \sigma}{\partial x} & =0 & & \text { (momentum) } \\
\frac{\partial c}{\partial t} & =k \theta \frac{\partial^{2} \mu}{\partial x^{2}} & & \text { (diffusion) } \\
\frac{\partial}{\partial t}\left[\frac{1}{2} v^{2}+\Phi\right]-\frac{\partial}{\partial x}[v \sigma] & =\nu \frac{\partial^{2} \theta}{\partial x^{2}} & & \text { (energy) }
\end{aligned}
$$

Received June 22, 1999.

2000 Mathematics Subject Classification. Primary 35B35, 35B40, 35Q72. 
with the following notation being introduced:

$$
\begin{aligned}
w & \text { displacement } \\
v & \text { velocity } \\
\varepsilon & \text { strain } \\
\sigma & \text { stress } \\
\theta & \text { temperature } \\
\eta & \text { entropy } \\
c & \text { concentration } \\
\mu & \text { chemical potential } \\
\Phi & \text { internal energy } \\
\psi & \text { free energy. }
\end{aligned}
$$

For a thermo-mechano-chemical system we will make the constitutive assumption that the internal energy depends on either set of state variables $(\varepsilon, \eta, c)$ or $(\varepsilon, \theta, \mu)$. With state variables $(\varepsilon, \eta, c)$ :

$$
\begin{aligned}
\Phi & =\widehat{\Phi}(\varepsilon, \eta, c), \\
\sigma & =\widehat{\Phi}_{\varepsilon}(\varepsilon, \eta, c)=\hat{\sigma}(\varepsilon, \eta, c), \\
\theta & =\widehat{\Phi}_{\eta}(\varepsilon, \eta, c)=\hat{\theta}(\varepsilon, \eta, c), \\
\mu & =\widehat{\Phi}_{c}(\varepsilon, \eta, c)=\hat{\mu}(\varepsilon, \eta, c)
\end{aligned}
$$

or, with state variables $(\varepsilon, \theta, \mu)$ :

$$
\begin{aligned}
\Phi & =\Phi(\varepsilon, \theta, \mu), \\
\psi & =\Phi-\theta \eta-\mu c=\psi(\varepsilon, \theta, \mu), \\
\sigma & =\psi_{\varepsilon}(\varepsilon, \theta, \mu)=\sigma(\varepsilon, \theta, \mu), \\
\eta & =\psi_{\theta}(\varepsilon, \theta, \mu)=\eta(\varepsilon, \theta, \mu), \\
c & =\psi_{\mu}(\varepsilon, \theta, \mu)=c(\varepsilon, \theta, \mu),
\end{aligned}
$$

where subscripts denote differentiation with respect to that variable.

The following relations are obtained by differentiation:

$$
\begin{aligned}
& \Phi_{\theta}=\psi_{\theta}+\eta+\theta \eta_{\theta}+\mu c_{\theta}=\theta \eta_{\theta}+\mu c_{\theta}, \\
& \Phi_{\mu}=\psi_{\mu}+\theta \eta_{\mu}+c+\mu c_{\mu}=\theta \eta_{\mu}+\mu c_{\mu}, \\
& \Phi_{\varepsilon}=\psi_{\varepsilon}+\theta \eta_{\varepsilon}+\mu c_{\varepsilon}=\sigma+\theta \eta_{\varepsilon}+\mu c_{\varepsilon} .
\end{aligned}
$$

From (5), (6), and (7) and subsequent differentiation it follows that

$$
\begin{aligned}
\sigma_{\varepsilon} & =\hat{\sigma}_{\varepsilon}+\hat{\sigma}_{\eta} \eta_{\varepsilon}+\hat{\sigma}_{c} c_{\varepsilon}, \\
\sigma_{\theta} & =\hat{\sigma}_{\eta} \eta_{\theta}+\hat{\sigma}_{c} c_{\theta}, \\
\sigma_{\mu} & =\hat{\sigma}_{\eta} \eta_{\mu}+\hat{\sigma}_{c} c_{\mu},
\end{aligned}
$$


which combine to give the following relationship between the "moduli":

$$
\hat{\sigma}_{\varepsilon}=\sigma_{\varepsilon}-\frac{c_{\mu} \eta_{\varepsilon}-c_{\varepsilon} \eta_{\mu}}{c_{\varepsilon} \eta_{\theta}-c_{\theta} \eta_{\mu}} \sigma_{\theta}-\frac{c_{\varepsilon} \eta_{\theta}-c_{\theta} \eta_{\varepsilon}}{c_{\varepsilon} \eta_{\mu}-c_{\theta} \eta_{\mu}} \sigma_{\mu}
$$

From equations (2), (3), and (4) we obtain the reduced field equations

$$
\begin{aligned}
\frac{\partial^{2} w}{\partial t^{2}} & =\frac{\partial \sigma}{\partial x} \\
\frac{\partial c}{\partial t} & =k \theta \frac{\partial^{2} \mu}{\partial x^{2}} \\
\theta \frac{\partial \eta}{\partial t}+\mu \frac{\partial c}{\partial t} & =\nu \frac{\partial^{2} \theta}{\partial x^{2}}
\end{aligned}
$$

or, equivalently, reduce to

$$
\begin{aligned}
\frac{\partial^{2} w}{\partial t^{2}} & =\frac{\partial \sigma}{\partial x}, \\
\frac{\partial c}{\partial t} & =k \theta \frac{\partial^{2} \mu}{\partial x^{2}}, \\
\frac{\partial \eta}{\partial t} & =\frac{\nu}{\theta} \frac{\partial^{2} \theta}{\partial x^{2}}-k \mu \frac{\partial^{2} \mu}{\partial x^{2}} .
\end{aligned}
$$

It should be noted that in view of the above equations the Clausius-Duhem inequality [7] is automatically satisfied, i.e.,

$$
\frac{\partial \eta}{\partial t}+\frac{\partial}{\partial x}\left[-\frac{\nu}{\theta} \frac{\partial \theta}{\partial x}+k \mu \frac{\partial \mu}{\partial x}\right]=\frac{\nu}{\theta^{2}}\left(\frac{\partial \theta}{\partial x}\right)^{2}+k\left(\frac{\partial \mu}{\partial x}\right)^{2}>0 .
$$

By integration of (12) it follows that

$$
\left.\int \eta d x\right|_{\infty}-\left.\int \eta d x\right|_{0}=\int_{0}^{\infty} \int\left[\frac{\nu}{\theta^{2}}\left(\frac{\partial \theta}{\partial x}\right)^{2}+k\left(\frac{\partial \mu}{\partial x}\right)^{2}\right] d x d t<\infty
$$

For the right-hand side of (13) to be bounded, one should expect $\frac{\partial \theta}{\partial x} \rightarrow 0$ and $\frac{\partial \mu}{\partial x} \rightarrow 0$ as $t=\infty$, which implies that the system tends to the state $\theta=$ constant and $\mu=$ constant as $t \rightarrow \infty$.

III. Hadamard instability of the linearized system. We next study sufficient conditions for instability of the thermo-mechano-chemical system (2), (3), and (4). By linearization of equation (11) about a constant state $(\varepsilon, \theta, \mu)$ we obtain the system

$$
\begin{aligned}
& \frac{\partial^{2} w}{\partial t^{2}}=\sigma_{\varepsilon} \frac{\partial^{2} w}{\partial x^{2}}+\sigma_{\theta} \frac{\partial \theta}{\partial x}+\sigma_{\mu} \frac{\partial \mu}{\partial x} \\
& c_{\varepsilon} \frac{\partial^{2} w}{\partial x \partial t}+c_{\theta} \frac{\partial \theta}{\partial t}+c_{\mu} \frac{\partial \mu}{\partial t}=k \theta \frac{\partial^{2} \mu}{\partial x^{2}}, \\
& \eta_{\varepsilon} \frac{\partial^{2} w}{\partial x \partial t}+\eta_{\theta} \frac{\partial \theta}{\partial t}+\eta_{\mu} \frac{\partial \mu}{\partial t}=\frac{\nu}{\theta} \frac{\partial^{2} \theta}{\partial x^{2}}-k \mu \frac{\partial^{2} \varpi}{\partial x^{2}} .
\end{aligned}
$$


In order to investigate Hadamard-type instability of the system, we try solutions of the form

$$
\begin{aligned}
& w(x, t)=a e^{p x} e^{\lambda^{2} t}, \\
& \mu(x, t)=b p e^{p x} e^{\lambda^{2} t}, \\
& \theta(x, t)=g p e^{p x} e^{\lambda^{2} t} .
\end{aligned}
$$

Setting $\xi \equiv p^{2} / \lambda^{2}$, instability will follow if $\lambda \gg 1$ implies $\xi<0$, in which case bounded initial data will yield solutions exponentially increasing at any time $t$. Substituting (15) into (14) yields the homogeneous system for $a, b, g$ :

$$
\begin{aligned}
& \lambda^{2} a=\xi \sigma_{\varepsilon} a+\xi \sigma_{\mu} b+\xi \sigma_{\theta} g \Rightarrow a=\frac{\xi}{\lambda^{2}-\sigma_{\varepsilon} \xi}\left[\sigma_{\mu} b+\sigma_{\theta} g\right] \\
& c_{\varepsilon} a+c_{\mu} b+c_{\theta} g=k \theta \xi b \\
& \eta_{\varepsilon} a+\eta_{\mu} b+\eta_{\theta} g=\frac{\nu}{\theta} \xi g-k \mu \xi b .
\end{aligned}
$$

Setting the determinant of (16) to zero provides the relationship between $\lambda^{2}$ and $\xi$. In order to investigate the sign of $\xi$ we distinguish the following cases:

Case $1 . k=0, \nu=0$. In this case equations $(16)_{2}$ and $(16)_{3}$ reduce to

$$
\begin{aligned}
\left(c_{\mu} \eta_{\theta}-c_{\theta} \eta_{\mu}\right) b & =-\left(c_{\varepsilon} \eta_{\theta}-c_{\varepsilon} \eta_{\varepsilon}\right) a, \\
\left(c_{\mu} \eta_{\theta}-c_{\theta} \eta_{\mu}\right) g & =-\left(c_{\mu} \eta_{\varepsilon}-c_{\varepsilon} \eta_{\mu}\right) a,
\end{aligned}
$$

which combined with $(16)_{1}$ yield the following condition between $\lambda^{2}$ and $\xi$ :

$$
\xi\left\{\sigma_{\varepsilon}-\frac{\sigma_{\varepsilon} \eta_{\theta}-c_{\theta} \eta_{\varepsilon}}{c_{\mu} \eta_{\theta}-c_{\theta} \eta_{\mu}} \sigma_{\mu}-\frac{c_{\mu} \eta_{\varepsilon}-c_{\varepsilon} \eta_{\mu}}{c_{\mu} \eta_{\theta}-c_{\theta} \eta_{\mu}} \sigma_{\theta}\right\}=\lambda^{2} .
$$

In view of $(9),(18)$ is written as

$$
\xi \hat{\sigma}_{\varepsilon}=\lambda^{2}
$$

and hence,

$$
\begin{aligned}
& \hat{\sigma}_{\varepsilon}>0 \text { implies stability } \\
& \hat{\sigma}_{\varepsilon}<0 \text { implies instability. }
\end{aligned}
$$

Thus, for vanishing coefficient of diffusion and heat conductivity, the elastic modulus at constant entropy and concentration controls stability. This is the extension of the condition for stability of a thermo-mechano-chemical system at $k=0$, obtained recently by Markenscoff [8], where it was shown that the addition of diffusion may render the system unstable.

Case 2. $k>0$ and/or $\nu>0$. In this case, under the assumption $\lambda \gg 1$, in order to balance terms of the same order of magnitude in equation (16), there are two possibilities: either $\xi=O(1)$ or $\xi=O\left(\lambda^{2}\right)$, which are examined below. 
(a) Roots $\xi=O(1)$. In this case, from (16), it follows that $a=O\left(\lambda^{-2}\right)$ and the system of $(16)_{2}$ and $(16)_{3}$ reduces to

$$
\begin{aligned}
\left(c_{\mu}-k \theta \xi\right) b+c_{\theta} g & =0 \\
\left(\eta_{\mu}+k \mu \xi\right) b+\left(\eta_{\theta}-\frac{\nu}{\theta} \xi\right) g & =0
\end{aligned}
$$

with determinant

$$
k \nu \xi^{2}-[\underbrace{k\left(\theta \eta_{\theta}+\mu c_{\theta}\right)}_{\Phi_{\theta}}+\frac{\nu}{\theta} c_{\mu}]+\left(c_{\mu} \eta_{\theta}-c_{\theta} \eta_{\mu}\right)=0 .
$$

By virtue of $(7)$ and the last two equations $(6),(22)$ is written in the form:

$$
k \nu \xi^{2}-\left[k \Phi_{\theta}+\frac{\nu}{\theta} c_{\mu}\right]+\psi_{\mu \mu} \psi_{\theta \theta}-\psi_{\mu \theta}^{2}>0 .
$$

For stability it is sufficient that both roots $\xi$ be positive; for instability at least one root $\xi$ should be negative, that is,

$$
\left.\begin{array}{l}
\Phi_{\theta}>0 \\
c_{\mu}>0 \\
c_{\mu} \eta_{\theta}-c_{\theta} \eta_{\mu}=\psi_{\mu \mu} \psi_{\theta \theta}-\psi_{\mu \theta}^{2}>0
\end{array}\right\} \Rightarrow \text { stability }
$$

while an opposite sign in any one of the above equations implies instability.

Note here that $\Phi_{\theta}$, being the specific heat, is expected to be positive, and so is $c_{\mu} \equiv$ $-\psi_{\mu \mu}$. The conditions (24) express the convexity of the function $\psi(\varepsilon,-\theta,-\mu)$.

The instability when any one of (24) fails is of the Cahn-Hilliard spinoidal decomposition type. The "negative creep" instability will be obtained below in the treatment of the case where the roots $\xi$ are $O\left(\lambda^{2}\right)$.

(b) Roots $\xi=O\left(\lambda^{2}\right)$. Equations $(16)_{2}$ and $(16)_{3}$ reduce to

$$
\left(c_{\mu} \eta_{\varepsilon}-c_{\varepsilon} \eta_{\mu}\right) b+\left(c_{\theta} \eta_{\varepsilon}-c_{\theta} \eta_{\theta}\right) g=\xi\left\{k\left(\theta \eta_{\varepsilon}+\mu c_{\varepsilon}\right) b-\frac{\nu}{\theta} c_{\varepsilon} g\right\}
$$

and, for $\xi=O\left(\lambda^{2}\right)$, from (25) we must have

$$
\underbrace{k\left(\theta \eta_{\varepsilon}+\mu c_{\varepsilon}\right) b}_{\Phi_{\varepsilon}-\sigma}-\frac{\nu}{\theta} c_{\varepsilon} g \approx 0 .
$$

Depending on whether the coefficients $k$ and $\nu$ are zero or nonzero, we distinguish the following subcases:

(1): $\nu=0, k>0$.

$$
\begin{aligned}
b & \cong 0 \\
a & \cong \frac{\xi}{\lambda^{2}-\sigma_{\varepsilon} \xi} \sigma_{\theta} g, \\
c_{\varepsilon} \sigma_{\theta} \frac{\xi}{\lambda^{2}-\sigma_{\varepsilon} \xi} & =\frac{\theta\left(c_{\theta} \eta_{\varepsilon}-c_{\varepsilon} \eta_{\varepsilon}\right)-c_{\theta}\left(\theta \eta_{\varepsilon}+\mu c_{\varepsilon}\right)}{\theta \eta_{\varepsilon}+\mu c_{\varepsilon}}=\frac{c_{\varepsilon}\left(\theta \eta_{\theta}+\mu c_{\theta}\right)}{\theta \eta_{\varepsilon}+\mu c_{\varepsilon}},
\end{aligned}
$$


which yields

$$
-\{\sigma_{\varepsilon}-\overbrace{\overbrace{\theta \eta_{\varepsilon}+\mu c_{\varepsilon}}^{\Phi_{\varepsilon}-\sigma}}^{\underbrace{\theta \eta_{\theta}+\mu c_{\theta}}_{\Phi_{\theta}}} \frac{\sigma_{\theta}}{c_{\varepsilon}}\} \xi+\lambda^{2}=0
$$

and, in view of (6) and (7),

$$
-\left\{\sigma_{\varepsilon}-\frac{\Phi_{\varepsilon}-\sigma}{\Phi_{\theta}} \frac{\sigma_{\theta}}{c_{\varepsilon}}\right\} \xi+\lambda^{2}=0 .
$$

Hence, instability follows if

$$
\sigma_{\varepsilon}-\frac{\Phi_{\varepsilon}-\sigma}{\Phi_{\theta}} \frac{\sigma_{\theta}}{c_{\varepsilon}}<0
$$

(2): $\nu>0, k=0$.

The system (16) yields

$$
\begin{aligned}
& g \approx 0, \\
& a \cong \frac{\xi}{\lambda^{2}-\sigma_{\varepsilon} \xi} \sigma_{\mu} b-\left\{\sigma_{\varepsilon}-\frac{c_{\varepsilon}}{c_{\mu}} \sigma_{\mu}\right\} \xi+\lambda^{2}=0,
\end{aligned}
$$

from which instability occurs if

$$
\sigma_{\varepsilon}-\frac{c_{\varepsilon}}{c_{\mu}} \sigma_{\mu}<0
$$

(3): $\nu>0, k>0$.

The system (16) yields

$$
\begin{gathered}
g \cong \frac{k}{\nu} \frac{\theta}{c_{\varepsilon}}\left(\theta \eta_{\varepsilon}+\mu c_{\varepsilon}\right) b, \\
a \cong \frac{\xi}{\lambda^{2}-\sigma \xi_{\varepsilon}}\left\{\sigma_{\mu}+\frac{k}{\nu} \frac{\theta}{c_{\varepsilon}} \sigma_{\theta}\left[\theta \eta_{\varepsilon}+\mu c_{\varepsilon}\right]\right\} b, \\
\frac{\xi}{\lambda^{2}-\sigma \xi_{\varepsilon}}\left\{c_{\varepsilon} \sigma_{\mu}+\frac{k}{\nu} \theta \sigma_{\theta}\left[\theta \eta_{\varepsilon}+\mu c_{\varepsilon}\right]\right\}+c_{\mu}+\frac{k}{\nu} \frac{\theta}{c_{\varepsilon}} c_{\theta}\left[\theta \eta_{\varepsilon}+\mu c_{\varepsilon}\right]=k \theta \xi .
\end{gathered}
$$

Balancing the leading terms in equation (35) gives

$$
-\sigma_{\varepsilon} \xi+\lambda^{2}=0
$$

so that instability follows if

$$
\sigma_{\varepsilon}<0
$$

that is, if the modulus at constant temperature and chemical potential is negative. This is the instability condition called "negative creep" by $\mathrm{Li}[2]$.

In conclusion the instability analysis of the coupled system of partial differential equations and the ensuing analysis of the sign of the eigenvalues for a coupled thermomechano-chemical system has provided in a unified way not only the conditions for instability due to spinoidal decomposition, but also the conditions for instability due to "negative creep" type.

Acknowledgment. Discussions with Professor J. R. Rice are acknowledged. 


\section{REFERENCES}

[1] J. W. Cahn and John E. Hilliard, Free energy of a nonuniform system. I. Interfacial free energy, J. Chemical Phys. 28, 258-267 (1958)

[2] J. C. M. Li, Negative creep and mechanochemical spinoidal in amorphous metals, Materials Science and Engineering 98, 465-468 (1988)

[3] E. Girt, P. Tomic, I. Gazdic, and T. Mihac, The relaxation of $C u_{60} Z r_{40}$ metallic glass under tensile stress, J. Phys. F. 13, 747-751 (1983)

[4] P. T. Vianco, Creep and structural relaxation of amorphous metals, Ph.D. Thesis, University of Rochester, Rochester, NY, 1986

[5] R. M. Bowen, Toward a thermodynamics and mechanics of mixtures, Arch. Rat. Mech. Anal. 24, 370-403 (1967)

[6] F. C. Larché and J. W. Cahn, The interactions of composition and stress in crystalline solids, Acta Metall. 33, 331-357 (1985)

[7] I. Mueller, Thermodynamics, Pitman, London, 1985

[8] X. Markenscoff, Diffusion induced instability, Quart. Appl. Math. 59, 147-151 (2001) 